\title{
Novel histone deacetylase inhibitor N25 exerts anti-tumor effects and induces autophagy in human glioma cells by inhibiting HDAC3
}

\author{
Xin-Yuan Sun ${ }^{1}$, Yue Qu ${ }^{1,2}$, An-Ran Ni ${ }^{1}$, Gui-Xiang Wang ${ }^{1}$, Wei-Bin Huang ${ }^{1,4}$, Zhong- \\ Ping Chen ${ }^{2}$, Zhu-Fen Lv ${ }^{3}$, Song Zhang ${ }^{1,5}$, Holly Lindsay ${ }^{6}$, Sibo Zhao ${ }^{6}$, Xiao-Nan Li ${ }^{6}$ \\ and Bing-Hong Feng ${ }^{1}$

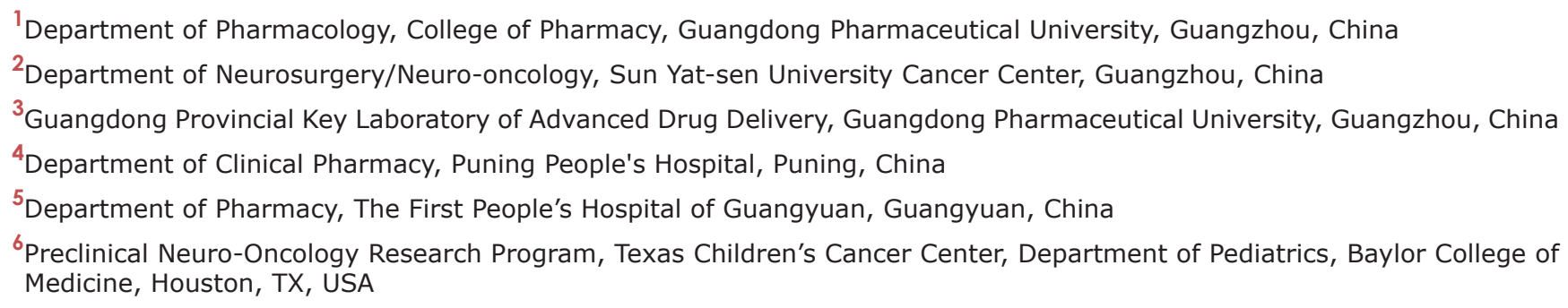 \\ Correspondence to: Bing-Hong Feng, email: fengbh2010@163.com \\ Keywords: histone deacetylase inhibitor, N25, autophagy, glioma, HDAC3 \\ Received: December 02, $2016 \quad$ Accepted: August 06, $2017 \quad$ Published: September 08, 2017 \\ Copyright: Sun et al. This is an open-access article distributed under the terms of the Creative Commons Attribution License 3.0 \\ (CC BY 3.0), which permits unrestricted use, distribution, and reproduction in any medium, provided the original author and source \\ are credited.
}

\section{ABSTRACT}

N25, a novel histone deacetylase inhibitor, was created through structural modification of suberoylanilide hydroxamic acid. To evaluate the anti-tumor activity of $\mathbf{N} 25$ and clarify its molecular mechanism of inducing autophagy in glioma cells, we investigated its in vitro anti-proliferative effect and in vivo anticancer effect. Moreover, we detected whether $\mathbf{N} 25$ induces autophagy in glioma cells by transmission electron microscope and analyzed the protein expression level of HDAC3, Tip60, LC3 in glioma samples by western blot. We additionally analyzed the protein expression level of HDAC3, Tip60, ULK1 (Atg1), and Beclin-1 (Atg6) after treatment with N25 in glioma cells. Our results showed that the anti-tumor activity of $\mathbf{N} 25$ in glioma cells is slightly stronger than SAHA both in vitro and in vivo. We found that N25 induced autophagy, and HDAC3 was significantly elevated and Tip60 and LC3 significantly decreased in glioma samples compared with normal brain tissues. Nevertheless, N25 inhibited HDAC3 and up-regulated the protein expression of Tip60, ULK1 (Atg1), and Beclin-1 (Atg6) after treatment of glioma cells with N25. In conclusion, these data suggest that N25 has striking anti-tumor activity in part due to inhibition of HDAC3. Additionally, N25 may induce autophagy through inhibiting HDAC3.

\section{INTRODUCTION}

Glioma is both the most common and lethal primary brain tumor which occurs in adults and children $[1,2]$. The median survival rate is only 13-16 months after standard therapy and more than $70 \%$ of glioblastoma patients die within two years of diagnosis [3,4]. Therefore, development of novel therapeutic drugs and new treatment strategies is essential for improving glioblastoma survival.

Epigenetic changes play a key role in the occurrence and development of cancer [5-7] and are a hot spot in international cancer research. Histone acetylation and deacetylation are important ways of epigenetic modifications [8]. Histone deacetylases function together 
with histone acetyltransferases to accurately control gene expression by altering nucleosome conformation, and affecting the stability of several large transcription factor complexes [9]. Numerous studies have confirmed that various human cancers are associated with HDACs overexpression and that histone acetylation imbalance caused by enhanced intra-tumoral HDACs activity affects the occurrence and development of cancer [10-12]. Therefore, HDACs are a promising target for cancer therapy.

Histone deacetylases comprise a family of 18 proteins in humans, consisting of class I proteins (HDAC1, 2, 3 and 8), class IIa proteins (HDAC4, 5, 7, and 9), class IIb proteins (HDAC6 and 10), class III proteins (sirtuins 1-7) and class IV proteins (HDAC11). These enzymes remove acetyl groups from lysine on histones and other proteins [13].

Histone deacetylase inhibitors (HDACi) represent a class of anticancer drugs. Suberoylanilide hydroxamic acid (SAHA), also known as vorinostat, is a synthetic hydroxamic acid that inhibits class I and II HDACs through binding of its hydroxamic acid group with a zinc atom at the bottom of the catalytic cavity, and causing histone acetylations within transcription factors [14, 15]. SAHA is the first drug of this type that has been approved by the US Food and Drug Administration for the treatment of cutaneous T-cell lymphoma [16]. N25 (N1-(2, 5-dimethoxyphenyl)-N8-hydroxyoctanediamide) was obtained through structural modification of SAHA and has been patented for several years. Previous studies demonstrated that N25 is a novel histone deacetylase inhibitor with stronger pharmacological activity than SAHA in several cancer cells [17].

HDAC3, a member of the HDACs family, has been reported to be expressed in almost all human tissues including brain, and usually is up-regulated in solid tumors [18]. A meta-analysis of a variety of human cancers revealed that HDAC3 is one of the most frequently upregulated genes in cancer cells [19]. Additionally, altered HDACs expressions is implicated in the pathogenesis of glioma [20].

It is generally known that induction of caspasedependent apoptosis (type I cell death) is a major mechanism by which most chemotherapeutic drugs and radiation kill tumor cells. In recent years, induction of autophagic cell death (type II cell death), one type of nonapoptotic programmed cell death, has been extensively studied [21].

Autophagy is a process of self-degradation of cellular components [22]. Autophagy is characterized by a cell without nuclear condensation during the process of cellular self-digestion in which cellular constituents are engulfed in specialized double-membrane structures called autophagosomes. Autophagosomes then fuse with lysosomes to form autolysosomes for degradation of their cargos and regeneration of nutrients [21, 23]. Microtubuleassociated protein 1 light chain 3 (LC3) is required for the elongation of autophagosomes. LC3 has two forms: LC3-I and LC3-II. LC3-II is the most reliable marker for quantification of the level of autophagy in cells, which increases due to the conversion of LC3-I to LC3-II during autophagy.

Autophagy has been proposed to have a dual role, either killing or protecting the cells, depending on the stage of the disease or the surrounding cellular environment [24]. Therefore, assessing the role of autophagy in a context-dependent manner is crucial, especially when considering if autophagy-targeting can be used as anticancer therapy.

ULK1 and Beclin-1 are homologous proteins of Atg1 and Atg6 in mammals, respectively. ULK1, which is required for autophagy, is directly acetylated and stimulated after activation of acetyltransferase Tip60 [25]. Additionally, ULK1 induces autophagy by phosphorylating Beclin-1 and activating VPS34 lipid kinase [26]. Another study showed that knockdown of HDAC 3 in cells could increase the level of acetylation of Tip60 [27]. Therefore, we proposed a hypothesis that N25 induces autophagy probably through a new mechanism of upregulating Tip60 by inhibiting HDAC3, causing upregulation of ULK1 (Atg1) and Beclin-1 (Atg6), and resulting in induction of glioma cell autophagy.

In this research, we studied the antitumor activity of $\mathrm{N} 25$ in vitro and in vivo. We also validated whether N25 induces autophagy and detected the changes of crucial proteins in glioma samples. More importantly, we conducted preliminary investigation on the molecular mechanism of N25 induced autophagy. Our study demonstrated that N25 has striking anti-tumor activity and was the first to show that HDACi induces autophagy through inhibiting HDAC3.

\section{RESULTS}

\section{N25, a novel HDACi, inhibits the cell viability in glioma cells}

To explore the anti-proliferative activity of N25 on glioma cells, U87-MG and U251 cells were chosen for analyses. Cells were exposed to gradually increasing concentrations of N25 for 48 hours. Subsequently, cell viabilities were detected with Cell Counting Kit-8. The growth-inhibitory curves of U87-MG and U251 were plotted (Figure 1B, 1C), and $\mathrm{IC}_{50}$ values were calculated. The IC50 of N25 and SAHA were $2.55 \mu \mathrm{M}$ and 3.58 $\mu \mathrm{M}$ respectively in U87-MG. The IC50 of N25 and SAHA were $5.63 \mu \mathrm{M}$ and $8.13 \mu \mathrm{M}$ respectively in $\mathrm{U} 251$. The growth-inhibitory curves demonstrated that N25 suppressed U87-MG and U251 viability more significantly than SAHA at relatively low concentration in vitro. 


\section{N25 suppresses tumor growth in a glioma xenograft tumor model}

To evaluate the anticancer effect of N25 in vivo, an anti-tumor study was performed using a nude mice xenograft model. $3-5 \times 10^{6}$ U87-MG cells were subcutaneously implanted into the right armpit of nude mice. Two weeks after implantation, mice bearing similar sizes of tumors were divided into five groups and there are 6 nude mice in each group. N25 at one of three doses $(24,48$, or $96 \mathrm{mg} / \mathrm{kg})$, SAHA $(96 \mathrm{mg} / \mathrm{kg})$ or Control $(0.9 \%$ $\mathrm{NaCl})$. After intragastric drug administration over two weeks, the nude mice were sacrificed and the xenografted tumors from treatment and control groups harvested, dissected and weighed. The average tumor volume of each treatment group was significantly lower than that of the control group on day 14. Tumor growth curve and therapeutic effects are shown (Figure 1D, Table 1). Significant tumor growth inhibition was observed in the N25 treatment group compared to the control group, with tumor inhibition rate of $68 \%$ in the $96 \mathrm{mg} / \mathrm{kg} \mathrm{N} 25$ group.

\section{N25 induces autophagy in glioma cells and xenograft tumor model}

Several studies have suggested that SAHA can induce autophagy in certain human tumors [21, 23, 28$30]$. We hypothesized that $\mathrm{N} 25$ can induce autophagy in

A<smiles>COc1ccc(OC)c(NC(=O)CCCCCCC(=O)NO)c1</smiles>

B
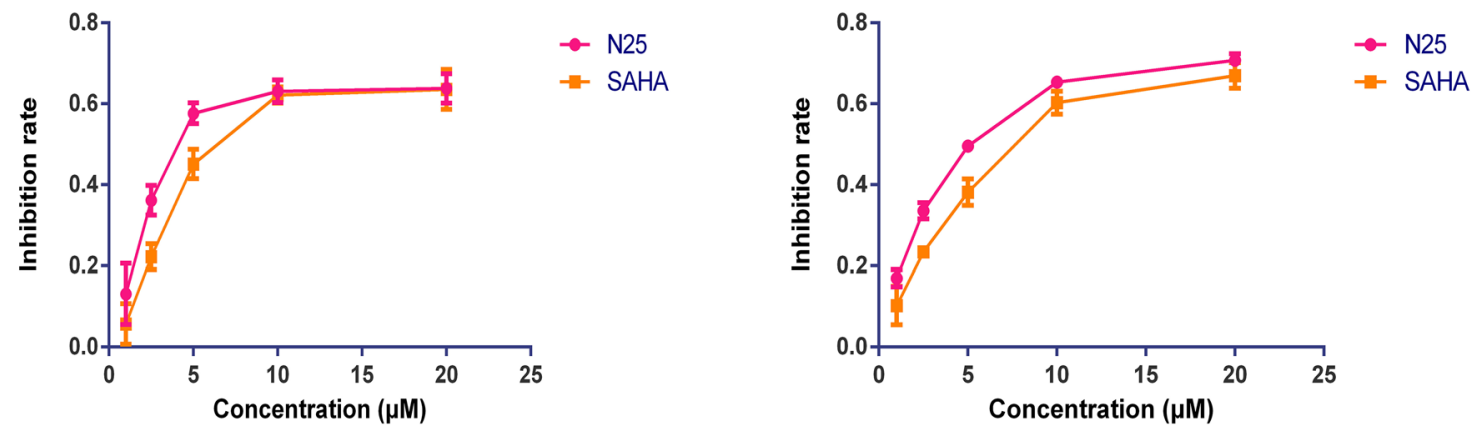

D
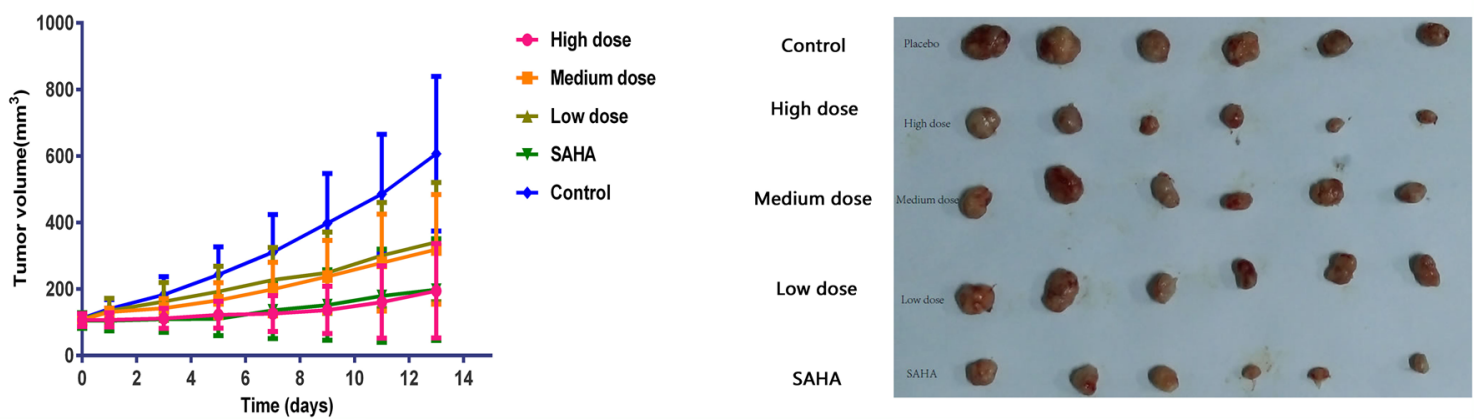

Figure 1: Anti-tumor effects of N25. (A) The chemical structure of N25. (B and C) U87-MG or U251 was exposed to gradually increasing concentrations of N25 or SAHA for 48 hours. Cell viability was detected by using the Cell Counting Kit-8. Cell growth was significantly inhibited after treatment with N25 in U87-MG and U251 cells ( $\mathrm{P}<0.05)$. (D) U87-MG was subcutaneously injected into nude mice. Mice were treated with one of three doses of N25, SAHA, or $0.9 \% \mathrm{NaCl}$. The tumor volume was measured $(\mathrm{P}<0.05)$. (E) Xenograft tumor picture. 
Table 1: The therapeutic effect of $\mathbf{N 2 5}$ in a U87 xenograft tumor model ( $n=6)$

\begin{tabular}{lccccc}
\hline \multirow{2}{*}{ Group } & Dose & \multicolumn{2}{c}{ Average weight } & & Tumor weight \\
\cline { 3 - 4 } & & $\mathbf{d} \pm$ d0 & $\mathbf{d 1 4}$ & TIR (\%) \\
\hline Control & $0.9 \% \mathrm{Nacl}$ & 20.1 & 19.5 & $0.93 \pm 0.36$ & 0 \\
High dose & $96 \mathrm{mg} / \mathrm{kg}$ & 20.2 & 18.8 & $0.30 \pm 0.24$ & $68 \% \pm 0.26^{* *}$ \\
Medium & $48 \mathrm{mg} / \mathrm{kg}$ & 20.1 & 19.2 & $0.52 \pm 0.26$ & $45 \% \pm 0.21^{*}$ \\
Low dose & $24 \mathrm{mg} / \mathrm{kg}$ & 19.8 & 19.1 & $0.60 \pm 0.30$ & $36 \% \pm 0.16^{*}$ \\
SAHA & $96 \mathrm{mg} / \mathrm{kg}$ & 19.9 & 19.2 & $0.32 \pm 0.21$ & $66 \% \pm 0.23^{* *}$ \\
\hline
\end{tabular}

Compared with control group, ${ }^{*} P<0.05,{ }^{* *} P<0.01$.

glioma cells, such as U87-MG. Transmission electron microscopy (TEM) is a standard method to reliably detect autophagy. After U87-MG was treated with N25 for 48 hours, TEM was used to assess morphological changes in N25 treated cells (Figure 2A). Numerous autophagosomes were present in N25-treated cells, whereas the formation of autophagosomes was not seen in control group. In order to further conform the hypothesis that $\mathrm{N} 25$ can cause autophagy in glioma cells, we performed western blot analysis. After treatment with $\mathrm{N} 25$, there was an accumulation of LC3II in U87-MG cells. Furthermore, we also demonstrated N25 can increase the expression of LC3 in xenograft tumor model by using immunohistochemistry (Figure $2 \mathrm{D}$ ), which suggest that N25 can induce autophagy in vivo. Overall, our results showed that $\mathrm{N} 25$ can induce autophagy in both glioma cells and xenograft tumor model.

\section{The expression of HDAC3, Tip60 and LC3 in glioma samples}

The expression of HDAC3, Tip60 and LC3 were measured in 20 glioma samples and 3 normal brain tissues. Western blot results revealed that the expression of HDAC3 was significantly higher in glioma samples than normal brain tissue, however, the expression of LC3 and Tip60 were lower compared with normal brain tissue (Figure 3A-3C).

\section{N25 inhibits HDAC3 and up-regulates Tip60}

To investigate the expression of HDAC 3 and Tip60 after treatment with N25 in glioma cells, western blot analysis was carried out. Results revealed that N25 significantly inhibits the expression of HDAC3 and upregulates the expression of Tip60 after treatment with N25 in U87-MG and U251 cells (Figure 4A, 4C).

\section{The expression of autophagy-related protein after N25 treatment}

Activated acetyltransferase Tip60 is known to directly acetylates and stimulates the protein kinase ULK1 [25]. ULK1 induces autophagy by phosphorylating Beclin-1and activating VPS34 lipid kinase [26]. To clarify whether N25 also activates ULK1 and Beclin-1 after Tip60 up-regulated by N25, we performed western blot analysis. The western blot results revealed that expression of ULK1 and Beclin-1 was significantly higher after treatment with N25 in U87-MG and U251 cells (Figure 4B, 4D).

\section{Selective HDAC3 inhibitor RGFP966 induces autophagy in glioma U87-MG cells}

To clarify N25 induces autophagy mainly associated with the inhibition of HDAC3, we applied a selective HDAC3 inhibitor RGFP966. Results showed that RGFP966 can increase the expression of LC3 in U87-MG cells (Figure 4E).

\section{DISCUSSION}

Glioma is the most common primary brain tumor which has the highest mortality rate. Most patients die within 2 years of diagnosis. Novel therapeutic drugs and new treatment strategies are urgently needed.

Overexpression of HDACs in tumor cells is associated with cell proliferation [31-35]. Knockdown of HDACs can induce a range of antitumor effects such as cell cycle arrest, inhibition of proliferation, induction of apoptosis, differentiation and senescence, and disruption of angiogenesis [36]. These data suggest that HDACs may be an effective target for anti-cancer drugs.

A recent study implicated that HDAC3 in the pathogenesis of pediatric glioma due to its promotion of 
A 2.5
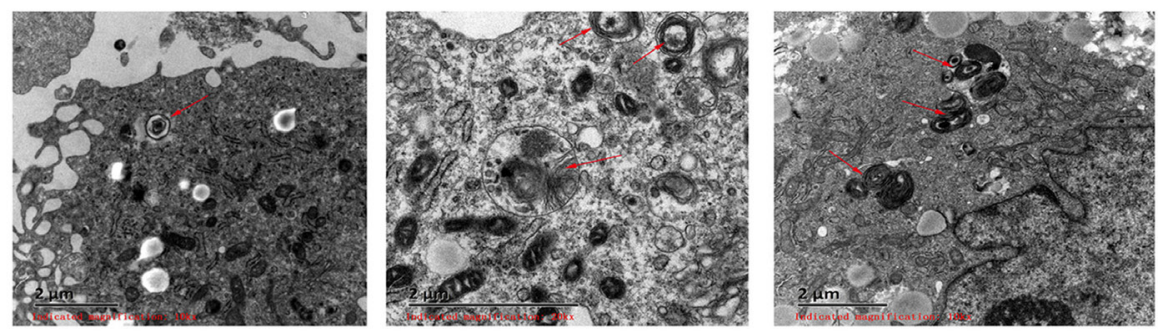

Control

SAHA
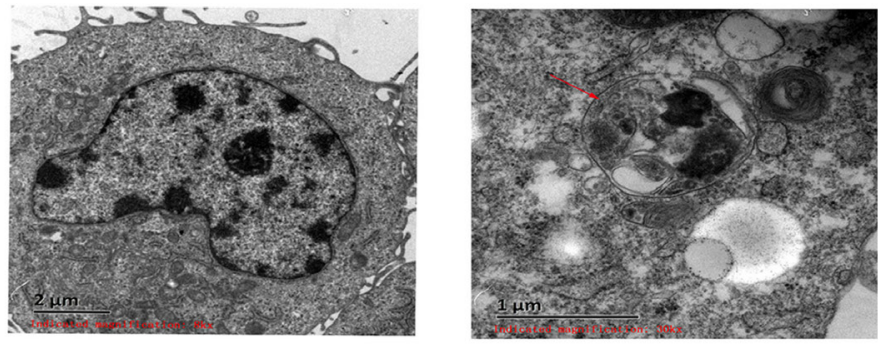

B
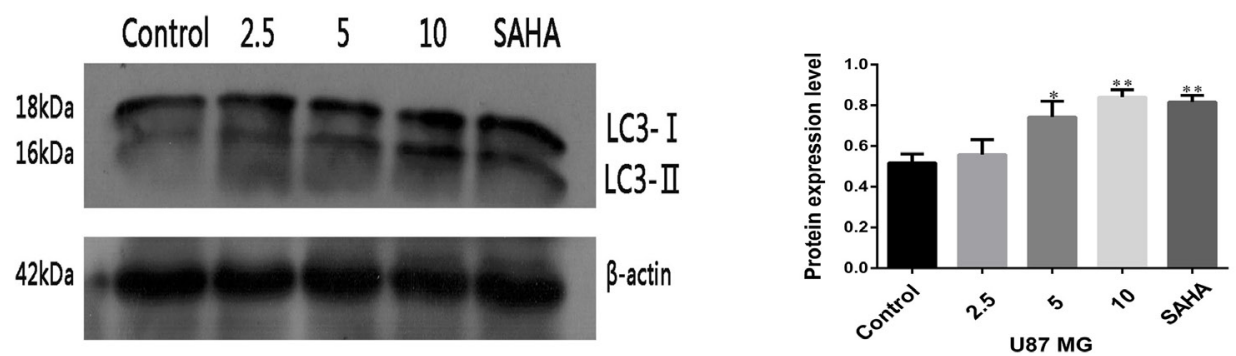

C
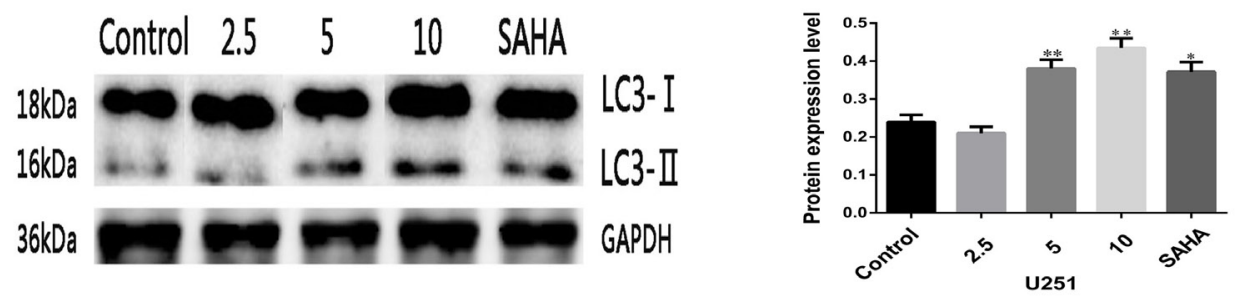

D
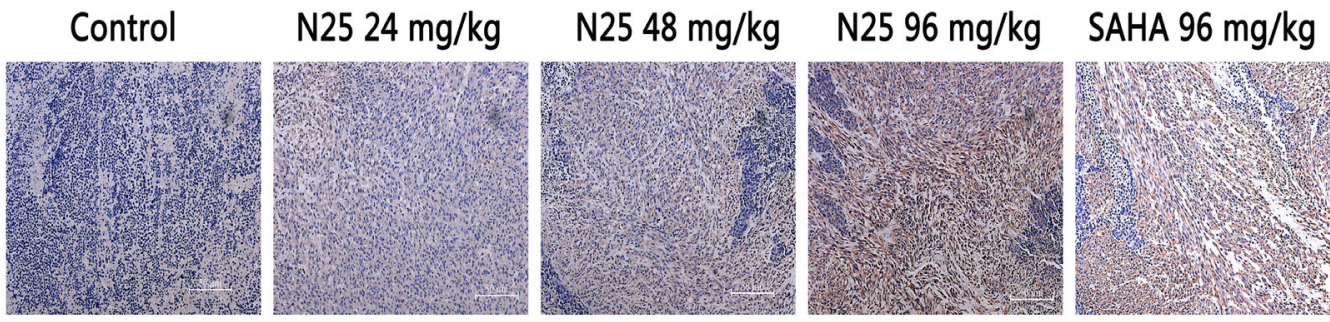

Figure 2: N25 induced autophagy in glioma cells and xenograft tumor model. (A) N25 induced autophagosome formation in U87-MG cells treated with various concentration $(2.5,5,10 \mu \mathrm{M})$ of $\mathrm{N} 25$ or $10 \mu \mathrm{M}$ SAHA for 48 hours. The red arrows present autophagosome. (B) N25 up-regulated protein expression of LC3-II in U87-MG cells. (C) N25 up-regulated protein expression of LC3-II in U251 cells. (D). N25 promoted LC3 protein expression in U87-MG xenograft tumor model. $* \mathrm{P}<0.05$ and $* * \mathrm{P}<0.01$ compared with control group. The data are shown as the mean $\pm \mathrm{SD}(\mathrm{n}=3)$, and representative results from three independent experiments with similar results are shown. 
glioma cell proliferation and migration, additionally, the level of HDAC3 expression was associated with tumor grades [37]. In order to identify whether HDAC3 is over-expressed in adult gliomas, we collected 20 patient samples and measured HDAC3 protein expression by western blot. Compared with normal brain tissues, glioma samples contained elevated expressions of the histone deacetylate HDAC3 but decreased levels of the histone acetyltransferase Tip60.
In order to explore the influence of N25 on HDAC3 and Tip60 expression in glioma cells, U87-MG and U251 cells were treated with N25. We determined that N25 inhibits HDAC3 expression and up-regulates Tip60.

To explore the in vitro anti-tumor activity of N25 in glioma cells, we conducted a cell proliferation assay. The results demonstrated that the anti-tumor activity of N25 in U87-MG and U251 cells are stronger than SAHA. We additionally established a U87-MG xenograft tumor model
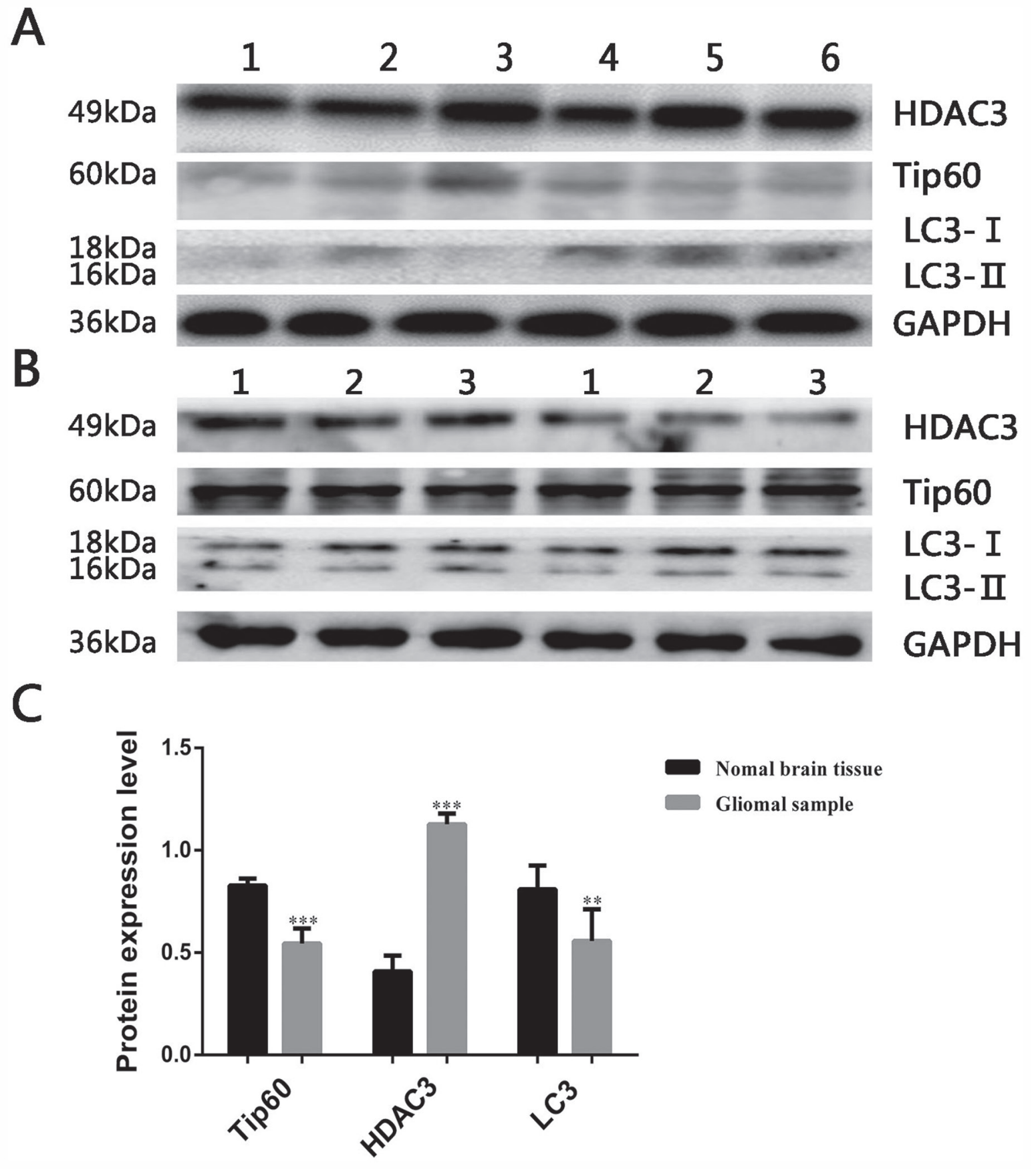

Figure 3: Expression of autophagy related protein in glioma samples. (A) Expression of HDAC3, Tip60 and LC3 in glioma samples, the blots have been cropped and retain 6 bands. (B) Expression of HDAC3, Tip60 and LC3 in 3 normal brain tissues. (C) Protein expression bar graph in glioma samples and normal brain tissues. $* * \mathrm{P}<0.01$ and $* * * \mathrm{P}<0.001$ compared with normal brain tissue. 
and confirmed that N25 equally exhibited significant antitumor activity in vivo.

In order to further study the role of autophagy in glioma cells. We determined that LC3 is expressed at much lower levels in glioma samples than in normal brain tissues. This suggests that low level of autophagy is beneficial to glioma growth. Next, to investigate whether
N25 induces glioma cells autophagy, we used TEM, a standard method to reliably detect autophagy, found that numerous autophagosomes were present in N25-treated cells. Further western blot experiment demonstrated that N25 $(5 \mu \mathrm{M})$ significantly increased endogenous LC3-II accumulation and induced autophagy.
A
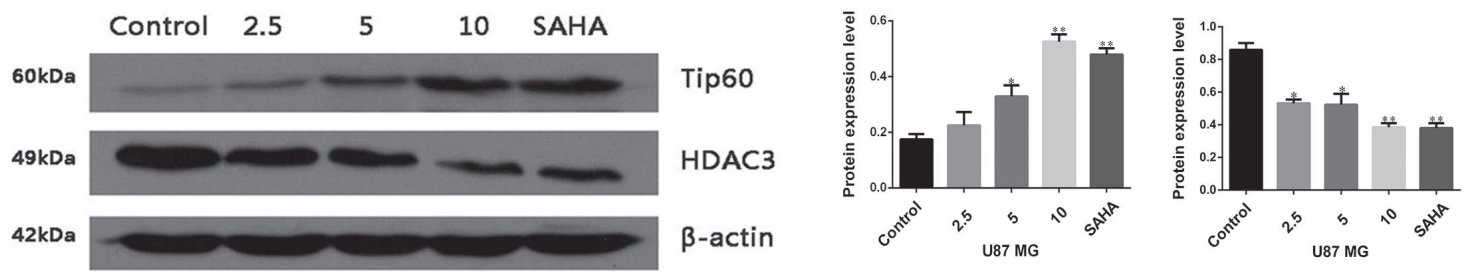

B

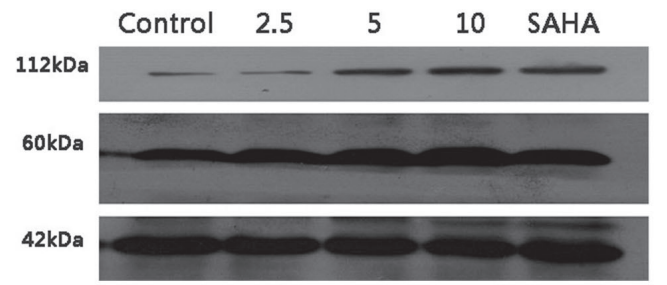

ULK1

Beclin-1

$\beta$-actin
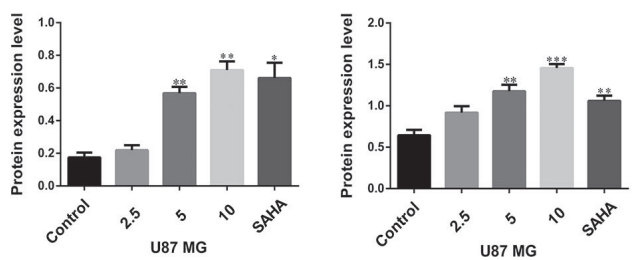

C
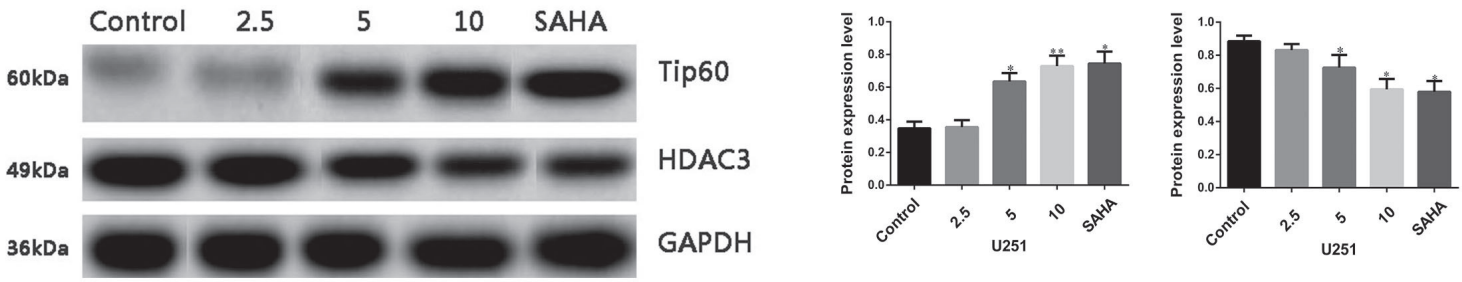

D
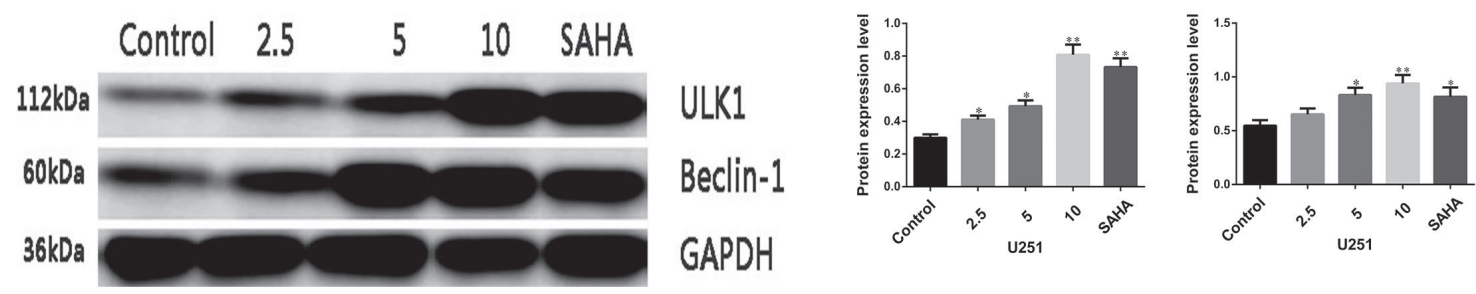

E

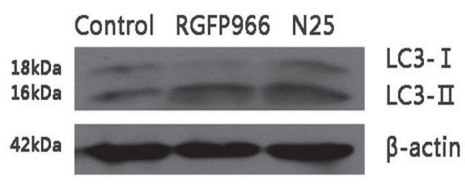

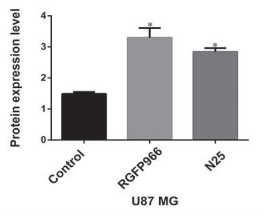

Figure 4: Protein expression after treatment with N25 in glioma cells. (A) Expression of HDAC3 and Tip60 after treatment with various concentration $(2.5,5,10 \mu \mathrm{M})$ of $\mathrm{N} 25$ or $10 \mu \mathrm{M}$ SAHA for 24 hours in U87-MG cells. (B) Expression of ULK1 and Beclin-1 after treatment with various concentration $(2.5,5,10 \mu \mathrm{M})$ of $\mathrm{N} 25$ or $10 \mu \mathrm{M}$ SAHA for 24 hours in U87-MG cells. (C) Expression of HDAC3 and Tip60 after treatment with various concentration $(2.5,5,10 \mu \mathrm{M})$ of $\mathrm{N} 25$ or $10 \mu \mathrm{M}$ SAHA for 24 hours in U251 cells. (D) Expression of ULK1 and Beclin-1 after treatment with various concentration $(2.5,5,10 \mu \mathrm{M})$ of N25 or $10 \mu \mathrm{M}$ SAHA for 24 hours in U251 cells. (E) Expression of LC3 after treatment with selective HDAC3 inhibitor RGFP966 (10 $\mu \mathrm{M})$ or N25 $(10 \mu \mathrm{M})$ for 24 hours in U87-MG cells. $* \mathrm{P}<0.05, * * \mathrm{P}<0.01$ and $* * * \mathrm{P}<0.001$ compared with control group. The data are shown as the mean $\pm \mathrm{SD}$ ( $\mathrm{n}=3$ ), and representative results from three independent experiments with similar results are shown. 
To examine whether N25 also activates ULK1 and Beclin-1 after up-regulated of Tip60 by N25, we performed western blot analysis. The western blot results revealed that expression of ULK1 and Beclin-1 was significantly higher after N25 treatment in U87-MG and U251. At last, to clarify N25 induces autophagy mainly associated with the inhibition of HDAC3, we applied a selective HDAC3 inhibitor RGFP966. The results showed that RGFP966 can increase significantly the expression of LC3 compared with control group in glioma U87-MG cells.

The study demonstrated that the anti-tumor activity of N25 in glioma cells is stronger than SAHA both in vitro and in vivo and may be mediated through induction of autophagy. Furthermore, HDAC3 was found to be significantly elevated and LC3 and Tip60 significantly decreased in glioma samples compared with normal brain tissues. Nevertheless, N25 inhibited HDAC3 and up-regulated the protein expression of Tip60, ULK1, and Beclin-1 in glioma cells. Meanwhile, after treatment with RGFP966 in glioma U87-MG cells, we found that autophagy induced by specific inhibition of HDAC3.

In conclusion, N25 has obvious anti-tumor activity in vivo and in vitro and through inhibition of HDAC3. N25 has slightly stronger activity than SAHA. N25 induces autophagy in glioma cells through a new mechanism of upregulating Tip60 by inhibiting HDAC3, causing upregulation of ULK1 (Atg1) and Beclin-1 (Atg6), and resulting in induction of glioma cell autophagy.

Through the research, we proposed and preliminarily demonstrated the possible mechanism of N25 induced autophagy in glioma cells on the basis of relevant reference. It was first proposed that HDACi induces glioma autophagy by inhibiting HDAC3. Meanwhile, this was the first time that we research the anti-tumor activity of HDACi N25 in vivo and the molecular mechanism of N25 induces glioma autophagy, and provided a possible direction for the treatment of glioma.

In this study, the results suggest that overexpression of HDAC3 in glioma cells is closely related to the level of autophagy. Other studies have confirmed that overexpression of HDACs is an important factor in tumorigenesis [10-12]. It also reported that HDAC3 overexpression is indeed exist in glioma and the proliferation of glioma cells was suppressed after knockdown of HDAC3 by siRNA [37]. Therefore, it is possible that overexpression of HDAC3 in glioma cells influences autophagy decrease and lead to glioma formation. As long as we changed the expression of HDAC3 in tumor cells and increase autophagy in glioma cells, it can lead to autophagic death of glioma cells. If glioma is this case, it can be used as a reference for glioma therapy that increasing autophagic cell death by inducing autophagy.

\section{MATERIALS AND METHODS}

\section{Cell culture}

Glioma cell line U87-MG and U251 were obtained from Sun Yat-Sen University Cancer Center. Cells were cultured in DMEM medium (Gibco, USA), supplemented with $10 \%$ fetal bovine serum at $37^{\circ} \mathrm{C}$ with $5 \% \mathrm{CO}_{2}$.

\section{Proliferation assay}

Cell Counting Kit-8 (yeasen, 40203ES80, China) was employed to determine the number of viable cells. In brief, $3 \times 10^{3}$ cells/well were seeded into 96-well plates and allowed to adhere. After treatment with various concentrations of N25 or SAHA for 48 hours, $10 \mu \mathrm{L}$ of CCK-8 solution was added to each well and the plates were incubated for another 2 hours. The absorbance was measured at $450 \mathrm{~nm}$ by Model 680 Microplate Reader (Bio-Rad, USA). The number of viable cells was counted by the absorbance. The value of IC 50 was calculated by SPSS 22.0.

\section{Reagents}

Vorinostat (SAHA, MK0683) was purchased from Dalian Meilun biotechnology. N25 were synthesized and identified according to the method in the literature [17]. RGFP966 (ApexBio, A8803, USA) was purchased from ApexBio.

\section{Antibodies}

Anti-LC3B antibody 1:3000 (Abcam, ab51520), Anti-HDAC3 antibody 1:4000 (Abcam, ab32369), Tip60 Antibody 1:1000 (Cell Signaling, \#12058), Beclin-1 Antibody 1:1000 (Cell Signaling, \#3738), and Anti-ULK1 antibody 1:5000 (Abcam, ab128859) were utilized for experiments.

\section{Transmission electron microscopy}

U87-MG cells were incubated with or without $100 \mathrm{mg} / \mathrm{mL}$ of PAMAM dendrimers G5 for 18 hours. Cells collected by trypsinization were fixed with $2.5 \%$ glutaraldehyde in $0.1 \mathrm{M} \mathrm{PBS}(\mathrm{pH} 7.4)$ for 2 hours at $4^{\circ} \mathrm{C}$ and washed three times with $0.1 \mathrm{M}$ cacodylate buffer containing $0.1 \% \mathrm{CaCl}_{2}$. Samples were post-fixed with $1 \%$ buffered osmium tetroxide ( $\mathrm{pH} 7.4$ ) for 30 minutes. After dehydration using graded ethanol, the samples were polymerized at $60^{\circ} \mathrm{C}$ for 48 hours, and cut into ultrathin sections. They were then stained with uranyl acetate and lead citrate, and finally examined using a JEM 1230 transmission electron microscope (JEOL USA, Inc.). 


\section{Western blot analysis}

The human glioma samples were obtained from Sun Yat-Sen University Cancer Center, and the relevant analysis of glioma samples were approved by the Ethics Committee of Sun Yat-Sen University Cancer Center. Glioma tissue or cells were lysed using RIPA lysis buffer (Beyotime, China) containing 1\% phenylmethanesulfonyl fluoride (PMSF) on ice for 30 minutes and then centrifuged at $12,000 \mathrm{RPM}$ at $4^{\circ} \mathrm{C}$ for 15 minutes. The supernatants were collected and total protein concentrations determined by using BCA protein assay kit (Pierce, Rockford, IL, USA). We chose $8 \%, 10 \%$ or $12 \%$ SDS-gels according to protein molecular weight. After separation by SDS-PAGE, the proteins were transferred to polyvinylidene difluoride (PVDF) membranes (Millipore, USA). The membranes were blocked in western blocking buffer (Beyotime, China) for 2 hours, rinsed with western washing liquid (Beyotime, China) for 1 minute and incubated with the pertinent antibody at $4{ }^{\circ} \mathrm{C}$ overnight. After washing with western washing liquid 3 times for 10 minutes, the membranes were incubated with horseradish peroxidase-conjugated secondary antibodies for 1 hour at room temperature. After development and fixing, the protein bands were analyzed by using Image $\mathrm{J}$ software, with $\beta$-actin or GAPDH as a control.

\section{Xenograft models}

Animal handling procedures were approved by the Institutional Animal Care and Use Committee of Guangdong pharmaceutical university. BALB/c-nu mice 4-5 weeks of age and were purchased from the experimental Animal Center of Sun Yat-Sen University. They were maintained under specific pathogen-free conditions. Subcutaneous xenografts were established by injection of $3-5 \times 10^{6}$ U87-MG cells per mouse to axilla ( $\mathrm{n}=6$ mice /group). Two weeks after inoculation, mice bearing similar size of tumors were randomly divided into five groups and then treated with one of three doses of N25 $(24,48$, or $96 \mathrm{mg} / \mathrm{kg})$, SAHA $(96 \mathrm{mg} / \mathrm{kg})$ or control $(0.9 \%$ $\mathrm{NaCl})$. Tumor diameter was measured by vernier caliper and tumor volume was calculated according to relevant formula. After intragastric administration of drug for two weeks, the nude mice were sacrificed and the xenograft tumors from all groups harvested, dissected, and weighed.

\section{Immunohistochemistry}

Specimens taken from the U87-MG xenografts were used for immunohistochemical detection of LC3. Sections of $4 \mu \mathrm{m}$ in thickness were deparaffinized and rehydrated with xylene and graded alcohol solutions. After washing with PBS, endogenous peroxidase activity was quenched by $3 \%$ hydrogen peroxide, and the sections were boiled in $10 \mathrm{mM}$ citrate buffer ( $\mathrm{pH}$ 6.0) for 3-5 minutes in an autoclave sterilizer followed by cooling at room temperature for more than 30 minutes. After rinsing with PBS, the sections were incubated with primary antibodies overnight at $4{ }^{\circ} \mathrm{C}$. Sections were stained with related antibodies, respectively. After rinsing with PBS, the sections were incubated with PV6001 or PV6002 for $1 \mathrm{~h}$ at $37^{\circ} \mathrm{C}$ and stained with DAB for 2 minutes. The slides were counterstained with hematoxylin, dehydrated with graded ethanol, cleared with xylene, and mounted in neutral gum. All slides were analyzed by two independent observers.

\section{Statistical analysis}

All values were presented as mean \pm SD. Statistical analysis is carried out by one-way ANOVA using the SPSS statistical software (SPSS 22.0). Probability values (P-value) $<0.05$ is considered as statistically significant.

\section{ACKNOWLEDGMENTS}

This study was supported by Science and Technology Planning Project of Guangdong Province (2013B021800079).

\section{CONFLICTS OF INTEREST}

The authors declare no conflicts of interest.

\section{REFERENCES}

1. Dunn GP, Rinne ML, Wykosky J, Genovese G, Quayle SN, Dunn IF, Agarwalla PK, Chheda MG, Campos B, Wang A, Brennan C, Ligon KL, Furnari F, et al. Emerging insights into the molecular and cellular basis of glioblastoma. Genes Dev. 2012; 26:756-784.

2. Filbin MG, Suva ML. Gliomas Genomics and Epigenomics: Arriving at the Start and Knowing It for the First Time. Annu Rev Pathol. 2016; 11:497-521.

3. Furnari FB, Fenton T, Bachoo RM, Mukasa A, Stommel JM, Stegh A, Hahn WC, Ligon KL, Louis DN, Brennan C, Chin L, DePinho RA, Cavenee WK. Malignant astrocytic glioma: genetics, biology, and paths to treatment. Genes Dev. 2007; 21:2683-2710.

4. Popescu AM, Purcaru SO, Alexandru O, Dricu A. New perspectives in glioblastoma antiangiogenic therapy. Contemp Oncol (Pozn). 2016; 20:109-118.

5. Sharma S, Kelly TK, Jones PA. Epigenetics in cancer. Carcinogenesis. 2010; 31:27-36.

6. Chi P, Allis CD, Wang GG. Covalent histone modifications - miswritten, misinterpreted and mis-erased in human cancers. Nat Rev Cancer. 2010; 10:457-469.

7. Kinnaird A, Zhao S, Wellen KE, Michelakis ED. Metabolic control of epigenetics in cancer. Nat Rev Cancer. 2016; 16:694-707. 
8. Hoshino I, Matsubara H. Recent advances in histone deacetylase targeted cancer therapy. Surg Today. 2010; 40:809-815.

9. Lu H, Yang XF, Tian XQ, Tang SL, Li LQ, Zhao S, Zheng HC. The in vitro and in vivo anti-tumor effects and molecular mechanisms of suberoylanilide hydroxamic acid (SAHA) and MG132 on the aggressive phenotypes of gastric cancer cells. Oncotarget. 2016; 7:56508-56525. https://doi.org/10.18632/oncotarget.10643.

10. Nakagawa M, Oda Y, Eguchi T, Aishima S, Yao T, Hosoi F, Basaki Y, Ono M, Kuwano M, Tanaka M, Tsuneyoshi M. Expression profile of class I histone deacetylases in human cancer tissues. Oncol Rep. 2007; 18:769-774.

11. Ishihama K, Yamakawa M, Semba S, Takeda H, Kawata S, Kimura S, Kimura W. Expression of HDAC1 and CBP/ p300 in human colorectal carcinomas. J Clin Pathol. 2007; 60:1205-1210.

12. Weichert W, Roske A, Gekeler V, Beckers T, Ebert MP, Pross M, Dietel M, Denkert C, Rocken C. Association of patterns of class I histone deacetylase expression with patient prognosis in gastric cancer: a retrospective analysis. Lancet Oncol. 2008; 9:139-148.

13. Falkenberg KJ, Johnstone RW. Histone deacetylases and their inhibitors in cancer, neurological diseases and immune disorders. Nat Rev Drug Discov. 2014; 13:673-691.

14. Marks PA. Discovery and development of SAHA as an anticancer agent. Oncogene. 2007; 26:1351-1356.

15. Codd R, Braich N, Liu J, Soe CZ, Pakchung AA. Zn(II)dependent histone deacetylase inhibitors: suberoylanilide hydroxamic acid and trichostatin A. Int J Biochem Cell Biol. 2009; 41:736-739.

16. Duvic M, Talpur R, Ni X, Zhang C, Hazarika P, Kelly C, Chiao JH, Reilly JF, Ricker JL, Richon VM, Frankel SR. Phase 2 trial of oral vorinostat (suberoylanilide hydroxamic acid, SAHA) for refractory cutaneous T-cell lymphoma (CTCL). Blood. 2007; 109:31-39.

17. Zhang S, Huang WB, Wu L, Wang LY, Ye LB, Feng BH. A novel suberoylanilide hydroxamic acid histone deacetylase inhibitor derivative, N25, exhibiting improved antitumor activity in both human U251 and H460 cells. Asian Pac J Cancer Prev. 2014; 15:4331-4338.

18. de Ruijter AJ, van Gennip AH, Caron HN, Kemp S, van Kuilenburg AB. Histone deacetylases (HDACs): characterization of the classical HDAC family. Biochem J. 2003; 370:737-749.

19. Pilarsky C, Wenzig M, Specht T, Saeger HD, Grutzmann R. Identification and validation of commonly overexpressed genes in solid tumors by comparison of microarray data. Neoplasia. 2004; 6:744-750.

20. Glozak MA, Seto E. Histone deacetylases and cancer. Oncogene. 2007; 26:5420-5432.

21. Chiao MT, Cheng WY, Yang YC, Shen CC, Ko JL. Suberoylanilide hydroxamic acid (SAHA) causes tumor growth slowdown and triggers autophagy in glioblastoma stem cells. Autophagy. 2013; 9:1509-1526.
22. He C, Klionsky DJ. Regulation mechanisms and signaling pathways of autophagy. Annu Rev Genet. 2009; 43:67-93.

23. Zhang J, Ng S, Wang J, Zhou J, Tan SH, Yang N, Lin Q, Xia D, Shen HM. Histone deacetylase inhibitors induce autophagy through FOXO1-dependent pathways. Autophagy. 2015; 11:629-642.

24. Hashimoto D, Ohmuraya M, Hirota M, Yamamoto A, Suyama K, Ida S, Okumura Y, Takahashi E, Kido H, Araki $\mathrm{K}$, Baba H, Mizushima N, Yamamura K. Involvement of autophagy in trypsinogen activation within the pancreatic acinar cells. J Cell Biol. 2008; 181:1065-1072.

25. Lin SY, Li TY, Liu Q, Zhang C, Li X, Chen Y, Zhang SM, Lian G, Liu Q, Ruan K, Wang Z, Zhang CS, Chien KY, et al. GSK3-TIP60-ULK1 signaling pathway links growth factor deprivation to autophagy. Science. 2012; 336:477-481.

26. Russell RC, Tian Y, Yuan H, Park HW, Chang YY, Kim J, Kim H, Neufeld TP, Dillin A, Guan KL. ULK1 induces autophagy by phosphorylating Beclin-1 and activating VPS34 lipid kinase. Nat Cell Biol. 2013; 15:741-750.

27. Yi J, Huang X, Yang Y, Zhu WG, Gu W, Luo J. Regulation of histone acetyltransferase TIP60 function by histone deacetylase 3. J Biol Chem. 2014; 289:33878-33886.

28. Yamamoto S, Tanaka K, Sakimura R, Okada T, Nakamura T, Li Y, Takasaki M, Nakabeppu Y, Iwamoto Y. Suberoylanilide hydroxamic acid (SAHA) induces apoptosis or autophagy-associated cell death in chondrosarcoma cell lines. Anticancer Res. 2008; 28:1585-1591.

29. Chen MY, Liao WS, Lu Z, Bornmann WG, Hennessey V, Washington MN, Rosner GL, Yu Y, Ahmed AA, Bast RC Jr. Decitabine and suberoylanilide hydroxamic acid (SAHA) inhibit growth of ovarian cancer cell lines and xenografts while inducing expression of imprinted tumor suppressor genes, apoptosis, G2/M arrest, and autophagy. Cancer. 2011; 117:4424-4438.

30. Frohlich LF, Mrakovcic M, Smole C, Zatloukal K. Molecular mechanism leading to SAHA-induced autophagy in tumor cells: evidence for a p53-dependent pathway. Cancer Cell Int. 2016; 16:68.

31. Halkidou K, Gaughan L, Cook S, Leung HY, Neal DE, Robson CN. Upregulation and nuclear recruitment of HDAC1 in hormone refractory prostate cancer. Prostate. 2004; 59:177-189.

32. Kawai H, Li H, Avraham S, Jiang S, Avraham HK. Overexpression of histone deacetylase HDAC1 modulates breast cancer progression by negative regulation of estrogen receptor alpha. Int J Cancer. 2003; 107:353-358.

33. Jung KH, Noh JH, Kim JK, Eun JW, Bae HJ, Xie HJ, Chang YG, Kim MG, Park H, Lee JY, Nam SW. HDAC2 overexpression confers oncogenic potential to human lung cancer cells by deregulating expression of apoptosis and cell cycle proteins. J Cell Biochem. 2012; 113:2167-2177.

34. Spurling CC, Godman CA, Noonan EJ, Rasmussen TP, Rosenberg DW, Giardina C. HDAC3 overexpression and colon cancer cell proliferation and differentiation. Mol Carcinog. 2008; 47:137-147. 
35. Muller BM, Jana L, Kasajima A, Lehmann A, Prinzler J, Budczies J, Winzer KJ, Dietel M, Weichert W, Denkert C. Differential expression of histone deacetylases HDAC1, 2 and 3 in human breast cancer-overexpression of HDAC2 and HDAC3 is associated with clinicopathological indicators of disease progression. BMC Cancer. 2013; 13:215.
36. West AC, Johnstone RW. New and emerging HDAC inhibitors for cancer treatment. J Clin Invest. 2014; 124:30-39.

37. Zhu J, Wan H, Xue C, Jiang T, Qian C, Zhang Y. Histone deacetylase 3 implicated in the pathogenesis of children glioma by promoting glioma cell proliferation and migration. Brain Res. 2013; 1520:15-22. 